\title{
Early complications of human bites to the hand in HIV-positive patients
}

\author{
Dr MTN Duma MBChB
}

Senior Registrar (pre-finals), Department of Orthopaedic Surgery

Dr LC Marais MBChB, FCS(Ortho), MMed(Ortho), CIME, PhD

HOD, Tumour Sepsis and Reconstruction Unit, Department of Orthopaedic Surgery

Grey's Hospital, Nelson R Mandela School of Medicine, University of KwaZulu-Natal, South Africa

\author{
Corresponding author: \\ Dr MTN Duma \\ Grey's Hospital \\ Town Bush Road \\ Pietermaritzburg 3201 \\ Email: mlekelelid@icloud.com \\ Cell: 0768195659
}

\begin{abstract}
Background: Human-inflicted bite wounds to the hand are serious injuries that may result in significant morbidity and permanent impairment. Irrespective of the mechanism, they are associated with a high complication rate and this has been attributed to the unique anatomy of the hand and the pathogens involved in human bites. HIV (human immunodeficiency virus) infection is known to compromise the immune system through immune exhaustion and senescence. This potentially increases the risk of complications following human bites to the hand in HIV-positive patients. The aim of this study was to determine if HIV infection is associated with an increased risk for the development of early complications following human bites to the hand.

Patients and methods: We retrospectively reviewed the records of a cohort of consecutive patients treated between June 2013 and October 2014. Patient charts were reviewed and information extracted with regard to demographics, the location and mechanism of the bite, occurrence of complications, HIV status and CD4 counts, as well as whether they were taking ARV (antiretroviral) medication at the time. The time from injury to presentation was recorded, as well as the time from injury to surgical intervention.
\end{abstract}

Results: Thirty-nine patients were included in the study and there were no exclusions. The mean age of patients was 31.5 years (range 14-60 years), with an approximately equal distribution between males and females. Sixteen patients $(41 \%)$ in our study cohort were found to be HIV-positive, 15 patients were HIV-negative (38\%), and in eight cases $(21 \%)$ the HIV status was unknown. Of the HIV-positive patients, $44 \%$ had CD4 counts below 350 cells $/ \mathrm{mm}^{3}$ but only four $(25 \%)$ were on ART (anti-retroviral therapy) at the time of injury. Complications occurred in $88 \%$ (14/16) of the HIV-positive patients compared to $80 \%(12 / 15)$ in the HIV-negative group (risk ratio $[R R]=1.09 ; 95 \%$ confidence interval $[\mathrm{CI}]=0.8-1.5 ; \mathrm{p}=0.65)$

Conclusions: HIV infection was not associated with an increased risk of developing complications following human bites to the hand in this series. An increase in the time from injury to presentation was however associated with an increased risk of amputation.

Key words: human bite, hand, HIV, complications, sepsis, infection

http:/ / dx.doi.org/10.17159/2309-8309/2016/v15n4a8

\section{Introduction}

Human-inflicted bite wounds to the hand are serious injuries that may carry grave consequences. This has been evident in medical literature since the nineteenth century. In 1870 a man's teeth marks were matched to his dead mistress and in 1894 a dentist died following a bite from a patient. ${ }^{1,2}$ In 1910 a case of a gangrenous paronychia was reported in a seven-year-old girl who had been a habitual nail-biter, and the first case of a clenched fist injury was reported in 1911. ${ }^{3}$ Surgical debridement has always formed an essential part of management, even during the earlier days where 'electrocautery and irrigation with fuming nitric acid' was a recognised method of treatment. ${ }^{3}$ 
Human bite injuries of the hand may be classified as either occlusive or clenched fist (fight bite). Irrespective of the mechanism, they are associated with a high complication rate and this has been attributed to the unique anatomy of the hand and the pathogens involved in human bites. ${ }^{3,4}$ In addition, wounds are generally small in size resulting in a casual approach to their management. About a third of patients who present with a human bite injury to the hand may present a different cause of their injury rather than a human bite and thus it is important to have a high index of suspicion when viewing injuries over the dorsal area of the

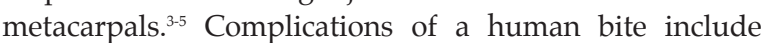
cellulitis, septic arthritis, tenosynovitis, hand stiffness, lymphangitis and osteomyelitis., 4,67

Certain developing regions have been experiencing a growing level of social and interpersonal violence. ${ }^{8}$ As a result, human bites to the hand have also been encountered more frequently. The increasing prevalence of this injury complex exposed the paucity of literature on the topic of human bites to the hand in HIV-positive patients. In the region where this study was performed an estimated $21.5 \%$ of adults between the ages of 15 and 50 years are living with HIV. ${ }^{9-11}$ HIV infection is known to compromise the immune system, through immune exhaustion and senescence. This potentially increases the risk of complications following human bites to the hand in HIV-positive patients.

The aim of this study was to determine if HIV infection is associated with an increased risk for the development of early complications following human bites to the hand.

\section{Patients and methods}

We retrospectively reviewed the records of a cohort of consecutive patients who were referred to our regional level orthopaedic unit with human bites of the hand between June 2013 and October 2014. Ethical approval was obtained from our institution's ethics review board prior to commencement of the research (BREC Ref: BE 020/15). Approval was also received from the provincial department of health and the hospital where the research was performed (Ref: HRKM 70/15).

All patients over the age of 13 years with human bite injuries to the hand were included. Cases where the human bite injury did not involve the hand or where the bite was not caused by a human were excluded. Patients with hand infection prior to suffering a human bite were also excluded. Patient charts were reviewed and information extracted with regard to demographics, the location of the bite, occurrence of complications, microscopy and culture results, antibiotic sensitivity profiles, HIV status of patients and their CD4 counts, as well as whether patients were on anti-retroviral medication at the time of presentation. The time from injury to presentation was recorded, as well as the time from injury to surgical intervention. Complications were defined as cellulitis, septic arthritis, tenosynovitis, osteomyelitis and surgical amputation.
Statistical analysis was performed using Stata 13.0 (StataCorp. College Station, Texas). Continuous variables were reported as mean $( \pm \mathrm{SD}$ ) or median (with interquartile range) and categorical variables as numbers and percentages, unless otherwise stated. Differences in continuous variables were compared with the use of the unpaired t-test or the Mann-Whitney test (depending on the distribution of the data). Categorical data was compared using the Fisher's exact test (if any cell count was below 5) or the chi-squared test (if no cell count below 5). All tests were two-sided and the level of significance was set at $\mathrm{p} \leq 0.05$.

\section{Results}

A total of 39 patients were included in the study and there were no exclusions. The mean age of patients was 31.5 years (range 14-60 years; standard deviation [SD] = 11.4 years), with an approximately equal distribution between males $(n=20)$ and females $(n=19)$. There was no significant difference in the age of patients by gender $(\mathrm{p}=0.48)$. The type of injuries were occlusional bites $(64 \%)$, fight bites $(15 \%)$, traumatic amputation $(18 \%)$ and one self-inflicted occlusion bite $(3 \%)$. The most commonly injured digit(s) were the thumb and ring finger ( $26 \%$ each), followed by the middle finger (18\%), with the remaining digits affected equally. Forty-three per cent of middle finger injuries were the result of a fight bite. In $8 \%$ of cases the anatomical location of the bite injury was not specified in the records. A total of 16 patients $(41 \%)$ in our study cohort were found to be HIVpositive, 15 patients were HIV-negative $(38 \%)$, and in eight cases $(21 \%)$ the HIV status was unknown. Of the HIV-positive patients, $44 \%$ had a CD4 counts below 350 cells $/ \mathrm{mm}^{3}$ but only four $(25 \%)$ were on ART (antiretroviral therapy) at the time of injury.

The mean time to presentation, time from injury to initial debridement and duration of hospital stay is given in Table I. The duration of hospital stay was found to be independent of the time to presentation $(\mathrm{p}<0.01)$ (Figure 1). The duration of hospital stay was significantly longer in the HIV-positive group (9.9 vs 4.7 days; $p<0.01$ ). None of the patients in our study group required admission to a critical care unit.

Complications occurred in $77 \%$ (30/39) of cases and the development of a complication was associated with an increased duration of hospital stay $(p=0.05)$ These included cellulitis $(n=17)$, soft tissue loss requiring split skin graft $(n=2)$, septic arthritis $(n=6)$, osteomyelitis $(n=3)$, tenosynovitis $(n=1)$ and surgical amputation $(n=5)$. There was no significant difference in the time to presentation (median $=3$ days, interquartile range [IQR] $=6$ ) of the group of patients who did develop complications in comparison to the group without complications (median = 1 day, IQR = 2) $(\mathrm{p}=0.07)$. We did, however, observe an increased time to presentation (median $=$ 7 days, IQR $=27)$ in the subset of patients who required amputation $(\mathrm{p}=0.05)$. 


\begin{tabular}{|c|c|c|c|c|}
\hline & $\begin{array}{c}\text { Overall } \\
(\mathrm{n}=39)\end{array}$ & $\begin{array}{l}\text { HIV-positive } \\
\quad(n=16)\end{array}$ & $\begin{array}{c}\text { HIV-negative } \\
\quad(\mathbf{n}=15)\end{array}$ & p-value \\
\hline Age (years) ${ }^{a}$ & $31.7 \pm 11.3$ & $37.8 \pm 10.7$ & $26 \pm 9.7$ & $<0.01$ \\
\hline Male gender (\%) & $20(51 \%)$ & 5 & 8 & \\
\hline Female gender $(\%)$ & $19(49 \%)$ & 11 & 7 & \\
\hline \multicolumn{5}{|l|}{ Type of injury } \\
\hline Occlusional bite $(\%)$ & $26(67 \%)$ & $13(81 \%)$ & $9(60 \%)$ & \multirow{3}{*}{0.46} \\
\hline Fight bite (\%) & $6(15 \%)$ & $1(6 \%)$ & $3(30 \%)$ & \\
\hline Traumatic amputation (\%) & $7(18 \%)$ & $2(13 \%)$ & $3(30 \%)$ & \\
\hline Time to presentation (days) & $5.7 \pm 10.4$ & $8.7 \pm 14.4$ & $2.5 \pm 3.9$ & 0.12 \\
\hline Time to 1st debridement (days) & $7.1 \pm 10.5$ & $9.8 \pm 14.4$ & $3.4 \pm 4.0$ & 0.10 \\
\hline Hospital stay (days) & $8.3 \pm 7.0$ & $9.9 \pm 6.5$ & $4.7 \pm 1.6$ & $<0.01$ \\
\hline Number of debridements & 41 & 16 & 15 & 0.69 \\
\hline Complications (\%) & $30(77 \%)$ & $14(88 \%)$ & $12(80 \%)$ & \multirow{6}{*}{0.65} \\
\hline Cellulitis & 23 & 9 & 10 & \\
\hline Septic arthritis & - & - & - & \\
\hline Osteomyelitis & 1 & - & 1 & \\
\hline Tenosynovitis & 1 & 1 & - & \\
\hline Amputation & 5 & 4 & 1 & \\
\hline
\end{tabular}

Complications occurred in $88 \%(14 / 16)$ of the HIVpositive patients compared to $80 \%(12 / 15)$ in the HIVnegative group (risk ratio $[R R]=1.09 ; 95 \%$ confidence interval $[\mathrm{CI}]=0.8-1.5 ; \mathrm{p}=0.65)$. When looking at major complications only, with the exclusion of cellulitis, the difference in the complication rate between the two groups remained insignificant $(p=0.39)$. Half of the HIV-positive patients who experienced complications had a CD4 below 350 cells $/ \mathrm{mm}^{3}$ and there was no difference in the complication rate in patients with a CD4 count above or below 350 cells $/ \mathrm{mm}^{3}(\mathrm{p}=0.48)$. The two HIV-positive patients who did not develop any complications had a CD4 count above 350 cells $/ \mathrm{mm}^{3}$. We found no relationship between ART and the development of complications $(p=1.0)$.

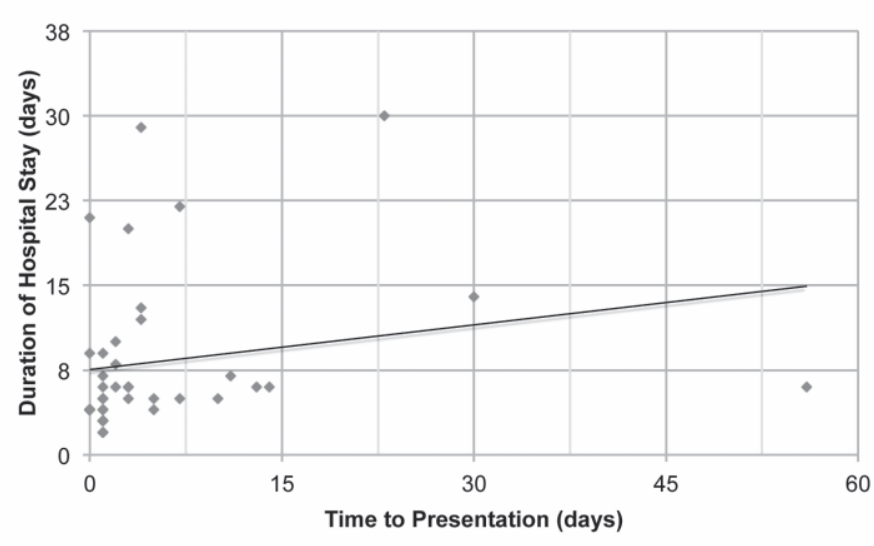

Figure 1. The duration of hospital stay was found to be independent of the time to presentation (Spearman's rank correlation coefficient $p=0.005$ )
Twelve HIV-positive patients $(75 \%)$ were not on ART at the time of the injury and $83 \%$ of these cases developed complications, while all of the patients $(n=4)$ whom were on ART developed complications. Four $(80 \%)$ of the patients requiring amputation were HIV-positive, although this was not statistically significant $(p=0.25)$. Two of the HIV- positive patients requiring amputation had a CD4 count below 350 cells $/ \mathrm{mm}^{3}$ and only one of these patients was receiving ART at the time of the injury.

Microscopy and culture results were only available in 11 cases. The most frequently cultured bacteria were Streptococcus spp $(\mathrm{n}=6)$ and Staphylococcus spp $(\mathrm{n}=5)$. These were followed by Bacillus spp $(\mathrm{n}=2)$, Enterococcus $(\mathrm{n}=1)$ and Klebsiella $(\mathrm{n}=1)$. Multiple organisms were identified in three cases. Eikenella corrodens was not identified in any of the cultures.

\section{Discussion}

Human bites involving the hand are frequently associated with the development of complications. ${ }^{4}$ Infectious complications are particularly common and may lead to permanent impairment as a result of soft tissue loss or partial or complete amputation of a digit or entire hand., , $^{4,12}$ Immune compromise has been suggested as a risk factor for an inferior outcome., ${ }^{72-14}$ However, limited data is available with regard to the influence of HIV infection on the development of complications in human hand bites. The aim of this study was to determine if patients living with HIV who experience a human bite to the hand are at increased risk for the development of early complications. Overall, complications occurred in $77 \%$ of cases. We found no difference in the rate of early complications in 
HIV-positive and -negative patients $(p=0.65)$. HIV-positive status was however associated with an increased duration of hospital stay $(\mathrm{p}=0.05)$.

The cases in our cohort were somewhat older (mean 31.5 years) than those in previous studies and we found an equal distribution of males and females, in comparison to other studies where mostly males were affected with an average age of 24 years. $3,7,8$ The most common type of human bite injury to the hand has previously been reported to be fight bites. $^{3.5}$ This was not our experience as $67 \%$ of cases involved the occlusive type of bite injuries. Delay in presentation has been documented extensively in the literature as the single most important determinant of morbidity following human bites to the hand. ${ }^{3,6,7,12,15,16}$ In our series, the association between time to presentation and the development of complications did not reach statistical significance $(p=0.07)$. There was however an increased risk of surgical amputation in cases with a delay in presentation $(\mathrm{p}=0.05)$.

There have been several recent clinical studies in orthopaedics that have shown that the HIV-positive population is not at an increased risk of infection or fracture non-union when compared to the HIV-negative population. Ferreira and Marais investigated the rate and severity of pin track sepsis in HIV-positive and HIV-negative patients treated with circular external fixators. In their retrospective analysis of 229 patients they found no statistically significant difference in the incidence of pin track sepsis $(p=0.94)$ and no statistically significant difference in the severity of pin track sepsis $(p=0.9)$ between the two groups. They also found that the CD4 count had no influence on either the incidence $(\mathrm{p}=0.57)$ or the severity $(\mathrm{p}=0.21)$ of pin track sepsis. ${ }^{10}$ Graham et al., in their prospective single blinded controlled study, presented $91 \mathrm{HIV}$-positive patients who underwent 103 procedures which involved the insertion of 111 implants. They followed up this group for a mean of 27 months, and found no association between the HIV status and developing late implant sepsis following internal fixation in acute trauma. They also found that if the HIV-positive patient develops early ( $<6$ weeks) infection and they receive appropriate management it is unlikely that they will develop late implant sepsis. ${ }^{17}$ Gardner et al. looked at fracture union following internal fixation in the HIV-positive population, in the largest study to date investigating fracture union in the HIV-positive population. Of 506 consecutive patients they had 96 HIV-positive patients with 107 factures that required internal fixation, with an average age of 37.8 years. They observed a non-union rate of $7.7 \%$ in femur fractures (the most frequent injury in their study) which was comparable to that found in series involving seronegative patients, whose non-union rate ranged from $0-10 \%$ in the femur. ${ }^{18}$

Immune compromise has been mentioned as a risk factor for the development of infection following human bites to the hand. ${ }^{7,12-15,19}$ Our data suggests that an HIV-positive patient is not necessarily at increased risk for the development of early complications, irrespective of anti-retroviral therapy or the patient's CD4 count. HIV-positive status was however associated with an increased duration of hospital stay $(p<0.01)$. Our data on the microbiology of infections was insufficient to draw any definitive conclusions in this regard. Previous studies identified Streptococcus spp and Staphylococcus spp, followed by Eikenella corrodens and anaerobes, as the most common organisms involved in human bites of the hand. ${ }^{5,720}$ We observed a similar trend, except we did not identify Eikenella corrodens in any of our specimens. Screening for exposure to hepatitis B virus was not performed in any of our patients. This is of concern as hepatitis B virus is $20-100$ times more likely to be transmitted from a human bite than HIV., ${ }^{714}$

We recognise the fact that this study has various limitations. The retrospective nature creates a problem in terms of the completeness of the data. This was illustrated by the fact that microscopy and culture results were only recorded in a small number of cases. A longer follow-up is required in order to assess the impact of HIV infection on a wider range of complications and permanent impairment. In addition the small difference in the frequency of complications in HIV-positive and -negative patients necessitates a much larger sample size. We propose that a larger prospective study is undertaken in order to determine the association of HIV infection and infectious complications as a result of human bites to the hand.

\section{Conclusion}

Patients with human bite injuries to the hand are at a high risk of developing complications. As such, they require urgent surgical debridement and administration of broad spectrum empiric antibiotic therapy that should be tailored to the suspected organism and adjusted according to the culture results and clinical response. In this series, HIV infection was not associated with an increased risk of developing complications following human bites to the hand. An increase in the time from injury to presentation was however associated with an increased risk of amputation.

\section{Compliance with ethics guidelines}

\section{- Conflict of interest}

Drs Duma and Marais have no financial or personal relationships with any individual and/or organisation that has or could have influenced their work.

This article is the sole work of the authors and no benefits have been received from a commercial entity related directly or indirectly to the subject of this article.

\section{- $\quad$ Ethics statement}

This article was not submitted to an ethical committee for approval.

Ethical approval was obtained from our institution's ethics review board prior to commencement of the research (BREC Ref: BE 020/15). Approval was also received from the provincial department of health and the hospital where the research was performed (Ref: HRKM 70/15). 


\section{References}

1. Pierce L, Strickland D. The case of Ohio vs Robinson. An 1870 bite mark case. Am J Forensic Med Pathol 1990;11(2):171-77.

2. Hyson JM Jr. Patient bites dentist: an 1894 case report. J Hist Dent 1998;46(2):76.

3. Tonta K, Kimble FW. Human bites of the hand: the Tasmanian experience. ANZ J Surg 2001;71(8):467-71.

4. Kennedy S, Stoll EL, Lauder AS. Human and other mammalian bite injuries of the hand: evaluation and management. J Am Acad Orthop Surg 2015;23(1):47-57.

5. Green DP, Wolfe SW, Hotchkiss RN, Pederson WC, Kozin SH. Green's operative hand surgery. Edn 6, Vol. 1, Elsevier Churchill Livingstone, USA, 2011, 73-74.

6. Dreyfuss UY, Singer M. Human bites of the hand: A study of one hundred six patients. J Hand Surg 1985;11:884-89.

7. Kelly IP. Management of human bite injuries of the hand. Injury 1996;27:481-84.

8. Website. [www.crimestatssa.com/precinct.php]. Visited on 08 August 2015.

9. Marais LC, Ferreira N. Osteosarcoma in adult patients living with HIV/AIDS. ISRN Onco. 2013 Mar 14; 2013: 219369. doi:10.1155/2013/219369.

10. Ferreira N, Marais LC. The effect of HIV infection on the incidence and severity of circular external fixator pin track sepsis: a retrospective comparative study of 229 patients. Strat Traum Limb Recon 2014;9:111-15.

11. Website. [http://www.health-e.org.za/wp-content/ uploads/2014/05/ASHIVHerp_Report2014_22May2014. pdf]. Visited 03 July 2015. The 2012 National Antenatal Sentinel HIV \& Herpes Simplex Type-2 Prevalence Survey in South Africa.

12. Harrison M. A 4-year review of human bite injuries presenting to emergency medicine and proposed evidence-based guidelines. Injury 2009;40:826-30.

13. Broder J, Jerrard D. Low risk of infection in selected human bites treated without antibiotics. Am J Emerg Med. 2004 Jan;22(1):10-13.

14. Morgan M. Hospital management of animal and human bites. J Hosp Infect 2005; 61:1-10.

15. Raval P, Khan W, Haddad B, Anant Narayan Mahapatra. Bite injuries to the hand - review of the literature. Open Orthop J 2014;8:204-208.

16. Mennen U, Howells CJ. Human fight-bite injuries of the hand. A study of 100 cases within 18 months. J Hand Surg Br 1991;16(4):431-35.

17. Graham SM, Bates J, Mkandawire N, Harrison WJ. Late implant sepsis after surgery in HIV positive patients. Injury 2015;46(4):580-84.

18. Gardner RO, Bates J, Ng'oma E, Harrison. Fracture union following internal fixation in the HIV population. Injury 2013;44(6):830-33.

19. Griego RD, Rosen T, Orengo IF, Wolf JE. Dog, cat, and human bites: a review. J Am Acad Dermatol 1995;33(6):1019-29.

20. Talan DA, Abrahamian FM, Moran GJ, et al. Clinical presentation and bacteriologic analysis of infected human bites in patients presenting to emergency departments. Clin Infect Dis 2003;37(11):1481-89.

This article is also available online on the SAOA website (www.saoa.org.za) and the SciELO website (www.scielo.org.za). Follow the directions on the Contents page of this journal to access it. 